\title{
ETHICS
}

\section{Do patients have duties?}

\section{H M Evans}

The notion of patients' duties has received periodic scholarly attention but remains overwhelmed by attention to the duties of healthcare professionals. In a previous paper the author argued that patients in publicly funded healthcare systems have a duty to participate in clinical research, arising from their debt to previous patients. Here the author proposes a greatly extended range of patients' duties grounding their moral force distinctively in the interests of contemporary and future patients, since medical treatment offered to one patient is always liable to be an opportunity cost (however justifiable) in terms of medical treatment needed by other patients. This generates both negative and positive duties. Ten duties-enjoining obligations ranging from participation in healthcare schemes to promoting one's own earliest recovery from illness-are proposed. The characteristics of these duties, including their basis, moral force, extent and enforceability, are considered. They are tested against a range of objections-principled, societal, epistemological and practical-and found to survive. Finally, the paper suggests that these duties could be thought to reinforce a regrettably adversarial characteristic, shared with rights-based approaches, and that a preferable alternative might be sought through the (here unexplored) notion of a "virtuous patient" contributing to a problem-solving partnership with the clinician. However, in defining and giving content to that partnership, there is a clear role for most, if not all, of the proposed duties; their value thus extends beyond the adversarial context in which they might first be thought to arise.

Correspondence to:

Professor H M Evans,

Centre for Arts and

Humanities in Health and

Medicine, School House,

Hild Lane, Durham;

h.m.evans@durham.ac.uk

Received 29 March 2007

Revised 27 June 2007

Accepted 27 June 2007
7 he reasonable duties and responsibilities of the patient are among the moody adolescents of the growing family of bioethics matters. They have emerged fairly late into daylight, are widely avoided in bioethics' daily business, are the object of a small number of admonitory interventions and have so far been successfully resistant to these while remaining persistently troublesome. In short, patients' duties clamour to be taken more seriously yet we continue to overlook them in favour of more "traditional" values attaching to the duties of the virtuous, but hard-pressed, healthcare professionals.

These moody adolescents have not been entirely ignored, of course. Commentators have already noticed the striking imbalance in the amounts of attention paid to doctors' duties and patient's responsibilities. ${ }^{1-3}$ Specific analyses have from time to time pointed out aspects of patients' unreasonable behaviours or expectations that imply correlative duties of self-restraint ${ }^{45}$ or have focused on the particular problem of the patient's failure to participate fully in consultation and management or to comply with treatment ${ }^{6}$ (notwithstanding the variety of reasons that might occasionally explain and excuse non-compliance ${ }^{7}$ ). In response, identifiable patients' duties have been proposed, variously grounded in taking seriously responsibility for error ${ }^{6}$, in taking autonomy seriously ${ }^{8} 9$, in putting limits upon autonomy in the interests of society ${ }^{10}$, in a naturalistic theory of ethics ${ }^{11}$ (itself hotly disputed ${ }^{12}$ ), in the fullness of a genuine therapeutic partnership between patient and professional ${ }^{4}{ }^{9}$ and in respect for the interests of significant other players including healthcare professionals $\mathrm{s}^{3}$, other patients in society ${ }^{3}$ and indeed the taxpayer. ${ }^{13}$

Nonetheless, the overall imbalance in scholarly and professional attention persists-extravagantly so, as a typical web-search will reveal-despite the best efforts of these thoughtful and scholarly interventions. My subsidiary intention in this paper is, therefore, to assert more stridently the patient's role in justly sought and justly acquitted healthcare interventions - to shout more loudly if previous voices have modestly been too muted.

However, my primary intention is to ground the moral tenability of these "patient obligations" in the interests of other patients, in contrast to the (usually) more subject-centred bases proposed by even the best and most subtle of earlier commentators. ${ }^{3}$ My reasons for this are threefold.

First, the broad therapeutic interests of patients collectively are the reason why publicly funded healthcare-in which context the "justice-based" responsibilities of patients most obviously arise-is there at all. Public provision of this sort involves mutual benefit and mutual participation: so my own medical treatment is, whatever else it is, liable to be an opportunity cost (however justifiable) in terms of the healthcare needs of others requiring comparable treatment at the time when I am treated. Both in general, and in the specific context of public healthcare provision, I have at least a prima facie moral responsibility to take other people's interests seriously; this implies that I ought not to incur opportunity costs to others avoidably, recklessly or excessively. (Of course I also have prudential reasons to promote that general responsibility, by contributing to it through my own actions.)

Second, and as a result, the interests of my "competitor" co-patients produce in me not merely the negative duties of avoiding either uncivil behaviour or needless waste, but also, provocatively, positive duties to promote my own health and, in the case of illness, to recover as quickly as possible; we shall see that this is a fairly red-blooded 
form of the proposed assertion of patients' responsibilities.

Third, objections to the arguments involved, objections that typically consist in appealing to the special vulnerability to which I am subject while I am ill, are immediately met by the overwhelmingly greater weight of just those same characteristics in the great mass of "competing" patients (whose concurrent treatment is an opportunity cost of my own treatment while I am receiving it, and whose healthcare needs are therefore most speedily attended to when my own needs are swiftly and efficiently out of the way). Such "sauce for the goose, sauce for the gander" replies are not the sole preserve of utilitarian perspectives, though they no doubt have an appeal there. My vulnerability when sick is, to say the least, given context by the vulnerability of these other patients; it would be better to say that my vulnerability is typically matched (if not out-matched) by theirs, considered in total. (Here I am following in a more general context the line of reasoning I earlier put forward in arguing for a more specialised duty of participation in clinical research. ${ }^{14}$ ) Let us now consider the case as it presents itself.

\section{THE CONTEXT}

To minimise the possibility of misunderstanding, let me specify as clearly as possible the context within which arise the particular duties that I will propose. They arise when-and, for my present purposes, only when-a competent, autonomous individual seeks healthcare from a publicly funded healthcare system that, in his particular society, he is eligible to enter regardless of whether he is at that time a taxpayer. (For grammatical convenience, and to make sure that at least one minority is properly represented in the argument, my patient is male throughout, and endures the pronoun "he".)

These duties may arise in other healthcare contexts as well, but I am not concerned to show that they do; I am concerned to show simply that they arise in a publicly funded system. Within this system, I shall take them to arise whether at the point of receiving care the patient pays nothing at all or pays a nominal or proportional charge. The point is that he does not pay the full economic cost of his care, or anything like it: he receives care that is wholly or partly subsidised from the collective purse.

\section{THE OSTENSIBLE DUTIES}

Let me now assert that, within the publicly funded healthcare context noted above, in seeking medical care a patient does in fact have, and should be recognised as having, at least the following 10 duties. Generally they relate to fairly self-evident "good things" which are in themselves not very surprising; but my claim is that these good things should form the content of obligations on the patient's part, their fulfilment being prima facie a condition of access to healthcare in the context described. Here are the duties.

\section{Duty to participate in a "healthcare jurisdiction"}

The patient should ensure that he is a recognised member of an institutional or social form of healthcare provision (what I will for the purposes of this paper call a "healthcare jurisdiction"). For instance, he should not live intentionally outside society's margins and then expect to be brought within the jurisdiction simply to get free healthcare. This is a conditional imperative only, of course: one has no absolute obligation to join the club, but membership of the club is a necessary condition of access to its benefits. Doubtless, in some jurisdictions it is not always easy to get into the clubi ${ }^{i}$; this proposed duty applies only where "entrance" is a realistic possibility and where a meaningful minimal threshold of public healthcare provision is available.

'I owe this objection to Neil Pickering.

\section{Duty to uphold his own health}

The patient should preserve and promote his own health and well-being so far as is reasonably open to him to do so. For instance, he should follow a responsible lifestyle that does not put his health avoidably at significant risk; he should follow health promotion guidelines; he should take account of the risk factors affecting him, including what he can reasonably know of his own family history. Questions obviously arise as to what "avoidably" here means. Occasionally a conflicting obligationsuch as to incur the risk of injury or disease-can arise in the course of responding to an emergency, or pursuing an occupation that carries above-average risks. The prima facie duty to avoid risk in general must therefore be subject to limitation by other, morally unavoidable, specific risks. Moral considerations frequently do, after all, conflict.

\section{Duty to protect the health of others}

The patient should avoid being a source of ill health for others within the same health jurisdiction. For instance, he should not allow himself to be an avoidable source of infection (one expression of Meyer's third duty ${ }^{9}$ ); he should not avoidably put at risk the health and safety of others (where "avoidably" may occasionally be subject to limitations similar to those just described); he should promote the health and well-being of his own dependants and he should not engage in, promote, or needlessly tolerate practices that are harmful to health.

\section{Duty to seek and access healthcare responsibly}

The patient should act responsibly regarding when and how he seeks healthcare. For instance, general practice surgeries already expect that patients with non-urgent conditions should accept the need to wait for an initial primary care appointment on a later day. If it is feasible for him to present himself at the surgery, he should not request a home visit. The patient also has a duty to be courteous and tolerant when attending healthcare institutions, including accident and emergency departments, as part of taking responsibility for the reasonableness of his own expectations of care. He should courteously respect all healthcare personnel, including non-clinical and administrative staff. (Comparable duties of responsible access are envisaged by Buetow ${ }^{5}$ and by Draper and Sorrell ${ }^{3}$.)

\section{Duty of truthfulness}

The patient should be truthful during the consultation and history-taking, thereby inter alia helping the achievement of a realistic diagnosis. (This duty resembles Meyer's first duty. ${ }^{9}$ ) For instance, he should divulge everything that is relevant, and he should avoid embellishment and evasion.

\section{Duty of compliance}

The patient should comply with his clinical management and medication unless he has good reason to think that these have not been properly arrived at (in which case he should declare that view and if necessary report it to competent authorities). Assuming that the patient has been properly involved in the decisions about his management and treatment, he should in general (and contrary to the limited defence of non-compliance offered by Conrad ${ }^{7}$ ) submit to any recommended investigations, take prescribed medication in the manner indicated and promptly report any adverse effects unless his condition is being closely monitored by others competent to do so.

\section{Duty of inpatient conduct}

If he is required to stay as an inpatient during the course of his treatment, the patient should be a responsible member of any inpatient community in which he finds himself. For instance, he should limit his noise and disturbance of others to the necessary minimum. I would even suggest that he should so far as possible avoid complaining (and should certainly avoid 
gossiping) to his fellow patients about any perceived shortcomings in his care, if this behaviour would discourage them and unjustifiably impede their relations with their own professional carers.

\section{Duty of recovery or maintenance}

During and after treatment, the patient should wherever possible promote his own recovery or, where this is unrealistic (for instance, in the case of chronic illness), try to maintain a reasonable quality of life. For instance he should try to ensure that he can convalesce in favourable conditions; he should comply with medical requests including attendance at repeat investigations and treatments and he should maintain and promote his own morale and that of any informal carers looking after him.

\section{Duty of research participation}

When certain strict conditions apply, chiefly concerning the therapeutic equivalence of all treatments he is likely to receive in this context, the patient should take part in clinical research relevant to his treatment when he is asked to do so. (I have explored this duty in detail elsewhere. ${ }^{14}$ )

\section{Duty of citizenship}

Finally, the patient should whenever the opportunity arises promote the healthcare jurisdiction that he has accessed-that is, appropriately support it beyond simply participating in it for the benefits of membership (as in duty no. 1). For instance, he should pay his relevant taxes in full and on time; and he should support only those political parties that credibly uphold the jurisdiction.

\section{CHARACTERISTICS OF THE DUTIES \\ Their basis}

It is now time to consider where these duties are grounded: they are frankly based in the common good and the common need. I am assuming (as do Draper and Sorrell ${ }^{3}$ and Ackroyd $^{13}$ - unlike, for example, Meyer $^{9}$ ) a patient who is a supplicant not merely for expertise but for a share of resources that are not merely scarce but are also commonly held (because publicly funded). My duties are proposed as part of the reasonable price of accessing them.

As such, their basis addresses the good of all other patients, first and foremost. In so doing, it addresses the individual patient's good as well, but partly as a means to the good of other patients and to the good of professional and informal carers and of others in society. Something like this is implicit in Parsons' coining of the "sick role", a conception that excused the patient from a number of ordinary duties such as the responsibility to turn up at work to earn a living and support his family, but replaced these ordinary duties with special ones attaching to the sick role itself. ${ }^{15}$ These included the duty to cooperate with the official agents of his care and recovery, something that aligns closely with my sixth, eighth and, implicitly, fifth duties.

In a nutshell, these duties (together with the "prophylactic" first, second and third duties) aim at making healthcare work most effectively and efficiently for the patient in question, optimising his recovery and hence indirectly the availability of other healthcare resources for other patients. Other duties promote wider courtesies (the fourth and seventh) or efficient research (the ninth), aiming directly at making healthcare work most effectively and efficiently for all patients.

\section{Their moral force}

To the extent that the wider good of other patients is important, then fulfilling these duties is important: that makes fulfilling them very important indeed. But they are conditional, not categorical. They are, as I have said, tied to the presumption that one wants to access publicly funded or subsidised healthcare. If you want something done for you (treatment provided to you, your recovery supported), then you should in return undertake correlative obligations such as those listed. Their moral force is therefore strong but conditional upon circumstance, including your being a competent and autonomous seeker after healthcare.

It is difficult to see why, in the abstract, one should be able to claim exemption from any of these duties. Hypothetically, if the duties are grounded in the interests of the wider common benefit (as I have argued), then if any are "negotiable" they are those that first protect the patient's own individual interests (such as the duties of being truthful and of compliance with treatment). Even here, though, wasted or misused resources ultimately threaten the interests of other healthcare users.

Those duties that directly protect the interests of others (such as the duty to seek healthcare responsibly, or to be a responsible member of an inpatient community) seem to me to be wholly non-negotiable. Nor, so far as I can see, are they lessened or mitigated in the long run by the acuteness or gravity of the patient's clinical need. His responsibilities regarding his own recovery seem to be rationally (self-interestedly) intensified in proportion to the gravity of his needs, and certainly morally intensified in proportion to the gravity of other potential patients' needs.

\section{Their extent}

It would be foolish and unworkable to pretend that the duties oblige the patient (intending or actual) to take every conceivable step to promote/uphold his health, to become exhaustively informed about treatments or to place himself in permanent quarantine so as to avoid all risk of ever infecting other people. However, this does not entail that there be no duty to attempt these things so far as is reasonable. The extent of what is reasonable will vary with circumstances and is a practical problem, rather than a conceptual flaw in the idea of duties such as these. Such problems are typical of duties carrying positive obligations (as distinct from the negative duties to refrain from proscribed activities), including, notably, the various positive duties of care and professionalism that are enthusiastically and unwearyingly imputed to clinical staff.

\section{Their enforceability}

Much the same sort of consideration applies to the duties' enforceability. If for practical purposes some are unenforceable, this does not defeat their prima facie applicability. We owe all sorts of unenforceable duties in daily life, such as those of respect and courtesy to others and, in a sense, of self-respect to ourselves with regard to our conduct in private.

Possibly none of my suggested duties is strictly enforceable, least of all perhaps the first three, which are what we might call the "prophylactic" duties of self-conduct prior to accessing healthcare (although coupled with various forms of health and safety legislation, the ban on smoking in public places-which could be exercised against anyone, patient or otherwiseenforces one specific aspect of the duty to protect the health of others). ${ }^{\text {ii }}$ The point is, rather, that the moral force of these duties should be acknowledged as applying to patients' attitudes and expectations, with consequent implications for their conduct. In due course, a world in which we believe we owe certain duties, even though they are not enforceable, will materially differ from a world in which we do not believe we owe those same duties.

ii I owe this point to Neil Pickering; like the UK, New Zealand has antismoking legislation of precisely this kind. 


\section{OBJECTIONS TO THE DUTIES}

It is now time to consider objections to these duties. These fall usefully into distinguishable categories.

\section{A pre-emptive objection}

I will begin with what might be called a "pre-emptive" objection to the whole discussion.

- Aren't most of these general duties that go without saying anyway?

In other words, do they really need to be stated ?ii $^{\text {Di }}$ Do they not apply to us in any case, in sickness and in health alike?

There are two responses to this. First, the need to state the duties (or something like them) arises from a significant imbalance in existing attention to the clinical context in which patients' rights and corresponding clinical duties hugely predominate over the responsibilities of patients themselves. They have gone too long without being stated, and neglect in stating them leads to neglect in living them, as evidenced by increases in widespread behaviour that is adverse to both health as such ${ }^{16}{ }^{17}$ and the security and effectiveness of healthcare provision. ${ }^{18} 19$

The objection might be pressed, however, that at least some of these duties (perhaps those of responsible accessing and of truthfulness) are duties that apply in other professional contexts as well. This might, indeed, be true, but it misses the point. One owes duties of truthfulness in other kinds of professional consultation typically in one's own interest; but all my suggested duties of the patient are grounded in the interests of others. These interests, moreover, are not the interests of clinical professionals per se in terms of their professional pride, autonomy and satisfaction, but, rather, are the interests of other patients via the effective use of scarce resources. (Those duties that do concern healthcare professionals' legitimate right to respect and civil discourse apply to them not by comparison with other kinds of professionals but rather by virtue of our duties of civility owed to all other citizens. The duties are ordinary and general in this sense, but they take their additional force in the healthcare context by contributing to a secure and effective healthcare provision in the interests of all.)

\section{Principled objections}

A second group of objections is grounded in the special gravity of healthcare need and in the vulnerability of the patient.

- Health is too important (significant, precious) to constrain healthcare in this way.

The objection here is that healthcare ought not to be made conditional upon the patient's fulfilling his duties, because there is something special about health and healthcare. It might run like this: "What you're talking about would be all very well if we were contemplating music lessons or sports coaching or even veterinary care for your pets, at the public's expense. If your non-cooperation made the service that we provided ineffective, then naturally we'd withdraw it. In the case of your pets, we'd probably have to arrange to have them removed from your care. But health isn't like that-it's just too important."

The right way to rebut this objection is to do it red-bloodedly. It is precisely this importance that applies to all other patients as well, actual and potential. It is precisely the special nature of their healthcare needs that requires responsible and effective use of resources held for the common good, and this in turn precisely requires of any individual patient his taking seriously the duties I have proposed.

iii I am grateful to Simon Walker for this objection.
- Most people can't choose anything other than this publicly funded route to healthcare.

It is true that privately funded healthcare (which for the sake of argument I have excluded from the scope of the proposed duties) is beyond the means of most patients, who, accordingly, have no choice but to opt in to the public system. For reasons that I have discussed elsewhere in connection with participation in clinical research ${ }^{14}$, this unfortunately entails no more than that those obliged to use a given system are also obliged to meet the conditions of using it. And we certainly believe this already. Nobody, I take it, seriously believes that one should be excused rude or threatening behaviour towards public healthcare staff simply because one is insufficiently wealthy to access alternative private systems. My other proposed duties are in this respect no more undermined by the constraints of opportunity than is the duty to be civil and peaceable.

Indeed, duties aimed at maximising the effectiveness of limited healthcare resources towards all become still more important if we recognise that most people are limited to accessing this form of healthcare provision. In this sense, the vulnerability of other patients is precisely what obliges any individual patient to behave responsibly.

\section{- Emergency care is typically not sought voluntarily}

Emergency care may involve the non-voluntary or even involuntary treatment of a patient. Such circumstances do indeed offer an inauspicious setting for that patient's duties; but since they do not meet the context that I set down at the outset, specifying an autonomous seeker after healthcare, they do not count against the argument given here for such duties.

- Seeking care as such is not wholly voluntary, since being ill is not voluntary

It is true that usually illness (or its consequences for dependants, carers, employers, and so on) is what obliges one to seek healthcare. But now the standard form of our response is becoming apparent: most other vulnerable patients are in precisely this situation, and you are still a supplicant seeking resources that they too need, in principle or in fact. Their vulnerability again matches your own so far as your duties as a patient are concerned. Again, we are not surprised by comparable constraints in other contexts. We do not choose to be dependent upon food and the other necessities of life; yet this is no reason for believing ourselves exempt from paying for them, nor even from queuing for them in a civil manner. In times of shortage we may also expect consumption to be done responsibly-something that rationing is intended to achieve, among other objectives.

\section{Objections on societal grounds}

A third form of objection emphasises the societal context in which individuals' choices are formed.

\section{- "Prophylactic" or "lifestyle" duties impose upon the victims of external factors without addressing those factors}

It is again true that there are structural influences on healthendangering behaviour or lifestyle, and we should certainly attend to those influences as another means of maximising the availability of healthcare resources. But the question is whether these influences dissolve the proposed duties, or discredit them as "victim-blaming". I do not believe they do for a momentthough not because I am at all discounting the societal context; indeed, I am insisting upon it in terms of the shared goods of public healthcare systems. 
Squarely put, not all who are subject to the influences of peer pressure, irresponsible tobacco and alcohol advertising or the ready availability of cheap "fast foods" in excessive quantities succumb to those influences. We can therefore certainly believe that those who do succumb ought not to, even if we may not in every case try to enforce against them every one of my proposed duties. The duties are still normative, even if not enforceable.

Again, parallels are instructive. Do we think that the presence of societal influences of greed, acquisitiveness, instant gratification, lure of materialism and so forth excuse shoplifting? Of course not. Do we think this should constrain our property laws or decriminalise shoplifting? Of course not. These influences offer explanations of why some people do certain things, but they are manifestly not justifications for doing them.

- Some non-compliance reflects cultural attitudes that should be respected

In general terms it is clearly right to respect the diversity of cultural attitudes and perspectives. My discussion implicitly presupposes a Western industrial cultural perspective in terms of the publicly funded healthcare context. Some attitudes attaching specifically to non-Western cultures, and some attitudes attaching to subgroups or folk beliefs within Western cultures, can be at odds with the conception of health underlying Western healthcare provision. For instance, within New Zealand's Western healthcare context, Maori culture admires large body mass-it is desirable to be big and, in effect, what would clinically be viewed as obese. ${ }^{20}$ Respect for this aspect of cultural diversity is difficult to reconcile with the duties necessary for optimising health. Presumably a culture that applauds large body size can nonetheless disvalue the blindness that sometimes follows diabetes, or the disabling effects of stroke, and individuals do indeed seek medical help for them. It is in the seeking of help that, I think, they can reasonably be expected to comply with concomitant duties. Urging compliance needs to be done sensitively, of course, but it is hardly respectful to the individual or his culture if we ignore the issue or pretend that no connection exists between a particular cultural norm and subsequent disease.

\section{Epistemological objections}

A fourth pair of objections concern the limitations of medical knowledge

\section{- Medical knowledge is indeterminate and provisional}

At first sight, the provisional (and fallible) nature of medical knowledge seems to limit-for instance-the duties of compliance with medical advice, since the requirement to comply is grounded in assuming that medical advice is good, and this assumption can be flawed. But on inspection this is not the objection it seems to be, since that very assumption surely underlies our seeking medical advice in the first place. We may accept that medical knowledge is revisable, but it's what we have now and it is the basis of what is on offer in the healthcare jurisdiction that is the context for my proposed duties. So, for instance, those who wish to hedge their bets by accessing both scientific and complementary medicine should do so responsibly. If the complementary therapy impairs the scientific ${ }^{21}$, then abandoning the complementary is a reasonable condition for continued access to the scientific.

\section{- Scientific medicine frequently gets it wrong}

This is the objection that the duty to comply with treatment takes insufficient account of the problem of adverse events; indeed it has been suggested that one in four hospital deaths results from suboptimal care. ${ }^{22}$ There is, of course, no duty to comply with dangerous treatment, but since the dangerousness of such treatment is almost never what the doctor intends ${ }^{\text {iv }}$, it provides us with a reason to make compliance more sophisticated and sensitive, frequently involving additional responsibilities on the part of the articulate patient to maintain accurate self-monitoring and clear communication with clinical staff. There should be no duty to endure net overall harms; but there should be a duty to take medication believed to be effective so long as that belief is rational.

\section{Practical objections}

Finally, we have a group of practical objections.

- Doctors don't actually know who complies and who doesn't; the duty cannot be monitored and hence cannot be enforced

This may be true; however, practical difficulties do not dissolve principled cases. We have already accepted the relative nonenforceability of most of the duties; their moral force, however, remains important and is, if anything, intensified by being a matter of personal responsibility and self-discipline on the part of patients.

- The duties would be unnecessary if healthcare resources were not scarce

If healthcare resources were abundant then the present grounds for a number of my proposed duties-the interests of other patients needing the same resources-would fall. Some duties (those of courtesy and, I think, participation in research) would remain. However, the other duties would retain force on other grounds. Responsible use of resources is intrinsically important and has ethical weight, even if we could materially afford to squander those particular resources: squandering seems wrong both morally and aesthetically, and it begets dangerous attitudes and habits that we can't afford elsewhere. Moreover, negligence with regard to one's health will produce other disbenefits in society beyond consuming healthcare resources.

- Compliance has costs too

A patient who fails to comply having already consumed consultation time represents an opportunity cost that must be reckoned heavily. But there can be a different opportunity cost when a patient complies-namely, the cost of the treatment consumed. While undeniably true, this objection seems to miss the point: properly targeting healthcare resources constitutes their proper use. When proper uses outweigh available resources, we have a situation of scarcity, but each proper use is a defensible opportunity cost, whereas avoidably ineffective or other improper uses are indefensible opportunity costs.

\section{AN INTERIM CONCLUSION}

I have identified a wide range of duties on the part of the would-be patient in a publicly supported healthcare system, I have grounded them distinctively in the welfare of other patients in general and I have defended them against a comparably wide range of categorised objections.

It might, however, be thought that I have answered one dispiriting proposition ("Doctors owe abundant duties!") merely with another ("And so do patients!"). The problem is, perhaps, the presumption that, short of a policeman in every consulting room, only the assertion of duties will keep either doctors or patients from reckless disregard of others' interests or selfish exploitation of the system for our own benefit. Can

${ }^{\text {iv }}$ Gross exceptions such as the notorious case of general practitioner $\mathrm{Dr}$ Harold Shipman are, thankfully, rare. 
we find no better moral basis for responsible conduct in healthcare? Can't the nature and potential gravity of healthcare needs call forth an ethic of altruism that overtakes prior contractual and other considerations?

There are two responses to this. First, such hopes are precisely what is called into question, or at least modified, by our noticing that patients' rights seem entirely to dominate and subdue any corresponding notion of their duties. Although there remains something adversarial about counterposing the duties of patients against those of the clinician, doing so at least redresses this dominance. Second, in most cases, the important (potentially) conflicting interests are not those of the clinician so much as those of other patients considered generally. Whenever I am not myself the index patient, I am of course among the group of those other patients. So for the moment, an ethic of enlightened self-interest will achieve in practical terms much of what we might hope for from an ethic of altruism, or at least the two will frequently align.

An alternative to this might consist in a kind of "problemsolving partnership" ${ }^{\prime \mathrm{v}}$, towards which both doctor and patient have responsibilities in making effective contributions to the partnership. But even if so, the notion of patients' duties remains substantial, through indicating the kinds of contributions that the patient has a duty to make. In particular, at least seven of my proposed duties (1, 2, 4, 5, 6, 8 and 9) would clearly make important contributions if acknowledged and fulfilled.

As a patient, I expect my doctor to fulfil her responsibilities towards me; she can reasonably expect the same from me. Our partnership will work if we have reasons to believe the responsibilities will be met. Here we meet a complication: if those reasons are grounded adversarially-for instance in the sanctions we can exert against one another-then the partnership may function but can hardly flourish. If, on the other hand, those reasons are grounded supportively-for instance in a framework of mutual trust - then the partnership can indeed flourish.

In this case, fulfilling my duties as a patient demonstrates my trustworthiness within the partnership. Furthermore, just as the virtuous doctor might be taken to be someone who through conviction and attitude is habitually moved to fulfil her responsibilities towards me, so it might be that the virtuous patient is one who through conviction and attitude is habitually moved to fulfil his responsibilities towards the doctor-each taking seriously the interests of the other, for the sake of the flourishing of the partnership and its instrumental effectiveness in upholding my health, and also for the sake of each other, tout court.

Although not all healthcare contexts will plausibly support such a high-flown moral framework, those that can-especially the longitudinal relationships found within primary care and chronic tertiary care-may also be supported by it. Such a framework will for some have the additional attraction of returning the moral focus to the individual patient, back from

${ }^{\vee}$ I am grateful to Grant Gillett for this suggestion. the totality of other (actual and potential) patients whose interests have been the bedrock of my arguments in this paper.

Here I can only suggest this alternative moral framework. A proper examination of the suggestion must be the subject of further work. However, in drawing attention to the basis, importance and plausible range of patients' duties, I hope to have reminded us already of the extent to which patients can and should contribute to the moral basis of healthcare-be it others' or their own.

\section{ACKNOWLEDGEMENTS}

This paper was developed during a period of sabbatical leave granted by Durham University, and it benefited from discussion with staff and postgraduate students at the Bioethics Centre, University of Otago, New Zealand, and at the Centre for Values, Ethics and Law in Medicine at the University of Sydney, Australia, courtesy of a University of Sydney Visiting Fellowship. I am grateful to all three universities. I am also grateful to two anonymous referees for their thoughtful and entirely helpful observations on the original manuscript.

Competing interests: The author is a member of the Royal College of General Practitioners' Committee on Medical Ethics and an Honorary member of the Governing Body of the Institute of Medical Ethics.

\section{REFERENCES}

1 Todd JS, Veatch RM. Medical ethics: A one-way covenant? Hastings Cent Rep 1980;10:4.

2 Wilson CL. Seeking a balance: Patient responsibilities in institutional health care. Med Law Int 1998;3:183-95.

3 Draper H, Sorell T. Patients' responsibilities in medical ethics. Bioethics 2002; 16:335-52.

4 Blustein J. Doing what the patient orders: maintaining integrity in the doctorpatient relationship. Bioethics 1993;7:290-314.

5 Buetow S. High need patients receiving targeted entitlements: what responsibilities do they have in primary health care? J Med Ethics 2005;31:304-6.

6 Buetow S, Elwyn G. Are patients morally responsible for their errors? J Med Ethics 2006:32:260-2.

7 Conrad P. The noncompliant patient in search of autonomy. Hastings Cent Rep 1987;17:15-7.

8 Madder $\mathrm{H}$. Existential autonomy: why patients should make their own choices. J Med Ethics 1997;23:221-5.

9 Meyer MJ. Patients' duties. J Med Philos 1992;17:541-55.

10 Buetow S. The scope for the involvement of patients in their consultations with health professionals: rights, responsibilities and preferences of patients. J Med Ethics 1998;24:243-7.

11 Sider RC, Clements CD. Patients' ethical obligation for their health. J Med Ethics 1984;10:138-42.

12 Gorovitz S. Why you don't owe it to yourself to seek health. J Med Ethics 1984;10:143-6.

13 Ackroyd DE. A rejection of doctors as moral guides. J Med Ethics 1984; 10:147

14 Evans HM. Should patients be allowed to veto their participation in clinical research? J Med Ethics 2004;30:198-203.

15 Parsons T. The social system. New York: Free Press, 1951.

16 Friedman JM. Obesity in the new millennium. Nature 2000;404:632-3.

17 House of Commons, Westminster Hall debate. Alcohol abuse and public order. HM Government: Weekly Hansard 200525 Jan.

18 Bleetman A, Fayeye OO. Preventing and managing violence and aggression in the NHS. Hospital Medicine 2003;65:728-31.

19 Peterson AM, McGhan WF. Pharmacoeconomic impact of non-compliance with statins. Pharmacoeconomics 2005;23:13-25.

20 Metcalf PA, Scragg RKR, Willougby $P$, et al. Ethnic differences in perceptions of body size in middle-aged European, Maori and Pacific people living in New Zealand. Int J Obes 2000;24:593-9.

21 Saunders J. Alternative, complementary, holistic. In: D Greaves, H Upton, eds. Philosophical problems in health care. Aldershot: Avebury, 1996:103-25.

22 Hayward RA, Hofer TP. Estimating hospital deaths due to medical errors. JAMA 2001;286:415-20. 